\title{
Older Women's Willingness to Discontinue Papanicolaou Test Screening
}

\author{
Ann Skelton, MD, Celine Kubn, MS, Debra Rothenberg, MD, and Alison Pope, MD
}

We surveyed our patients to assess their willingness to discontinue Papanicolaou testing. Our findings differ from those of previous studies, with $72 \%$ of our eligible respondents voicing willingness to discontinue screening. There may be many reasons for this, including linking the recommendation with the name of the patient's own physician. (J Am Board Fam Med 2014;27:295-296.)

The Papanicolaou test is a simple, effective screening tool for the detection of cervical cancer. There are now consistent recommendations by the US Preventive Services Task Force, the American College of Obstetrics and Gynecology, and the American Cancer Society (ACS) to discontinue screening most women at age 65 to $70 .^{1}$

The dramatic success of publicizing the benefits of screening and its role in reducing cervical cancer prevalence may have resulted in unwarranted patient eagerness for Papanicolaou tests. A national telephone survey about attitudes toward cancer screening found that only $43 \%$ of women would want to decrease the frequency of their Papanicolaou screening, even on the recommendation of their physicians. $^{2}$ A follow-up study found that $69 \%$ of surveyed women would continue annual Papanicolaou testing even if advised of the benefits of less intensive screening. ${ }^{3}$ Only 35\% of these women thought they would eventually stop screening, and of those, half thought they would not cease screening until age 80 .

\section{Methods}

This study examined elderly patients' receptiveness to the discontinuation of Papanicolaou testing. The

This article was externally peer reviewed.

Submitted 21 May 2013; revised 15 November 2013; accepted 25 November 2013.

From the Department of Family Medicine (AS, DR, AP), Maine Medical Center (CK), Portland, ME.

Funding: Support for this study was provided by the Maine Medical Center Medical Research Committee.

Conflict of interest: none declared.

Corresponding author: Ann Skelton, MD, 22 Bramhall St., Maine Medical Center, Portland, ME 04102 (E-mail: skelta@mmc.org). study received an exemption from the local institutional review board; consent was implicitly obtained by subject participation.

Participants in this study were patients of resident or attending physicians from 2 outpatient family medicine centers. Participants were women aged 70 or older who had had a Papanicolaou test within the past 3 years. They were identified from a laboratory database and review of electronic medical records. Exclusion criteria included cognitive impairment, hysterectomy, abnormal Papanicolaou tests within 10 years, previous cervical cancer or human papillomavirus infection, or incomplete or inaccurate information in the medical record.

Patients were contacted by phone and asked questions to determine whether they were eligible to discontinue screening based on ACS criteria. We asked eligible patients about their willingness to stop screening if their own named primary care physician advised them to do so. Of the 83 patients who completed the survey, 72 were eligible to stop Papanicolaou testing, and 52 $(72 \%)$ indicated a willingness to discontinue screening.

\section{Results}

Contrary to findings from previous studies, a large majority of our patients voiced willingness to discontinue screening. Many factors contribute to this difference, including geographic attitudes about medical care, degree of engagement in the medical system, and possible demographic and cultural differences. 


\section{Discussion}

We postulate that a key determinant in the greater acceptance of limited screening by our patients was the presentation of the national guidelines in the context of a recommendation from the physician regularly seen by the patient. In a letter to the editor written in response to the study by Schwartz et $\mathrm{al}^{2}{ }^{2}$ Schwenk ${ }^{4}$ commented on the importance of the quality and the trustworthiness of the patient's relationship with her physician in patient's acceptance of less frequent screening. He suggested that the authors missed this critical influence in the decisions women make to undergo cervical cancer screening.

National focus on delivering high-quality, costeffective care drives more limited use of testing where appropriate. Catalysts and barriers to patient acceptance of changing guidelines need to be iden- tified, especially when new recommendations contradict previously endorsed care. While our findings focus specifically on the topic of cervical cancer screening, we may be able to generalize the results supporting patient acceptance of limited screening to other tests.

\section{References}

1. Feldman S. Making sense of the new cervical-cancer screening guidelines. New Engl J Med 2011;365: $2145-7$.

2. Schwartz LM, Woloshin S, Fowler FJ, Jr., Welch HG. Enthusiasm for cancer screening in the United States. JAMA 2004;291:71-8.

3. Sirovich BE, Woloshin S, Schwartz LM. Screening for cervical cancer: will women accept less? AM J Med 2005;118:151-8.

4. Schwenk TL. Physician trust and cancer screening decisions. AM J Med 2005;118:1054. 